\title{
NUMERICAL INTEGRATION METHODS FOR THE SOLUTION OF SINGULAR INTEGRAL EQUATIONS*
}

\author{
BY \\ P. S. THEOCARIS ANI) N. I. IOAKIMII)IS \\ National Technical E'niversity, Athens
}

Summary. The evaluation of the stress intensity factors at the tips of a crack in a homogeneous isotropic and elastic medium may be achieved with higher accuracy and much less computation if the Lobatto-Chebyshev method of numerical solution of the corresponding system of singular integral equations is used instead of the method of Gauss-Chebyshev commonly applied to such problems. Comparison of results obtained by the two numerical methods when applied to the problem of a cruciform crack in an infinite medium proves the potentialities of the new approach.

1. Introduction. The problem of one or several cracks existing in an infinite, isotropic elastic medium and loaded in the same manner in both faces is reduced to the solution of a system of Cauchy-type singular integral equations by considering the cracks as composed of elementary dislocations placed infinitely close to each other. This system of singular integral equations is of the form:

$$
\sum_{i=1}^{N} c_{i j}(x) \int_{-1}^{1} \frac{\varphi_{j}(t)}{t-r} d t+\sum_{j=1}^{N} \int_{-1}^{1} K_{i j}(t, r) \varphi_{j}(t) d t=f_{i}(x), \quad i=1,2, \cdots, N,
$$

where functions $c_{i i}(x), K_{i j}(t, x)$ and $f_{i}(x)(i, j=1,2, \cdots, N)$ are known, while the unknown functions $\varphi_{i}(t)(j=1,2, \cdots, N)$ have singularities of order $(-1 / 2)$ as $t \rightarrow \pm 1$, that is at the tips of the cracks, and can be expressed in the form:

$$
\varphi_{j}(t)=\left(1-t^{2}\right)^{-1 / 2} g_{j}(t), \quad j=1,2, \cdots, N,
$$

with the functions $g_{i}(t)(j=1,2, \cdots, N)$ non-singular in the integration interval $[-1,1]$.

It should be noted that functions $\varphi_{i}(t)$ are directly related to the density of dislocations along the cracks and that the values of $g_{i}(t)$ for $t= \pm 1$ are proportional to the stress intensity factors at the crack tips $[1,2,3]$. The number $N$ of the singular integral equations in the system (1.1) is at maximum equal to double the number of the existing cracks if they are loaded with a normal and shear load.

Gol'dshtein and Savova [1] were the first who reduced the problem of a single, smooth and curved crack in an infinite isotropic elastic medium to a system of singular integral equations of the form (1.1), while the problem of several linear cracks arbitrarily oriented in an infinite isotropic elastic medium was solved by Datsyshin and Savruk [2]. Moreover, Erdogan and his co-workers have solved in a series of papers various other cases of linear cracks in half-planes or composite media. Some of these cases are contained in [3].

\footnotetext{
* Received February 25, 1976; revised version received Sept. 1, 1976.
} 
Furthermore, besides these cases of cracked plates, several other problems of mathematical physics may be reduced to systems of singular integral equations of the form (1.1) with unknown functions behaving like relations (1.2).

The simplest way of solving the system of singular integral equations (1.1) is by reducing it to a linear system of algebraic equations by approximating both singular and regular integrals by methods of numerical integration. Ergodan and Gupta [4] gave such a solution of the system (1.1) by developing a method of approximating the singular integrals identical with the Gauss-Chebyshev numerical integration method for ordinary integrals. Further, Erdogan, Gupta and Cook [3] extended this method for the case of unknown functions $\varphi_{i}(t)$ of the form:

$$
\varphi_{i}(t)=(1-t)^{\alpha}(1+t)^{\beta} g_{i}(t), \quad-1<\alpha, \quad \beta<1, \quad \alpha, \beta \neq 0 .
$$

where the functions $g_{i}(t)$ vary regularly in the integration interval.

However, the extension of the Gauss-Chebyshev method for the numerical determination of singular integrals and the solution of the corresponding systems of singular integral equations $[3,4]$ was not rigorous. In particular, the characterization of it as a Gauss method was not completely evident because it was proved to be accurate only for polynomials $g_{i}(t)$ up to the $(n-1)$ th degree ( $n$ is the number of the abscissae used), while the Gauss numerical integration method is accurate for polynomials up to the $(2 n-1)$ th degree included. It will be proved in the sequel that, indeed, the GaussChebyshev method applied to singular integrals, as developed by Erdogan and Gupta [4], is accurate for polynomials $g_{i}(t)$ up to $2 n$th degree included.

The Gauss-Chebyshev (or, generally, the Gauss-Jacobi) method was also extended for the numerical evaluation of singular integrals by Chawla and Ramakrishnan [5]. It will be shown later that their method applied to systems of singular integral equations of the form (1.1) is identical with the method developed by Erdogan, Gupta and Cook $[3,4]$.

By the Gauss-Chebyshev method, systems of the form (1.1) are reduced to systems of linear equations whose solution provides the values of the unknown functions $g_{i}(t)$ at discrete points $t_{k}(k=1,2, \cdots, n)$ within the integration interval $[-1,1]$, but not at the limits $t= \pm 1$. However, as already mentioned, stress intensity factors at the crack tips are proportional to the values of $g_{i}( \pm 1)$, which must therefore be evaluated from the values of $g_{i}\left(t_{k}\right)$, for example, by extrapolation. The relevant expressions are given by Krenk [6]. Nevertheless, additional error is introduced by such an extraploation, which is accurate only when the function $g_{i}(t)$ whose values at $t= \pm 1$ are to be determined is a polynomial up to $(n-1)$ th degree.

In order to avoid extrapolation error and the related computations, instead of the Gauss-Chebyshev method, the Lobatto-Chebyshev method can be used, which includes the points $t= \pm 1$ among the points $t_{k}(k=1,2, \cdots, n)$. In the present work, an extension of this latter method for singular integrals is developed in order to render it applicable to systems of the form (1.1). By applying both the Gauss-Chebyshev and the LobattoChebyshev methods in the evaluation of the stress intensity factor $k_{I}$ in a cruciform crack, the supremacy of the latter is demonstrated.

2. The Gauss-Chebyshev numerical integration method applied to singular integrals. The system of singular integral equations: 


$$
\begin{aligned}
\sum_{i=1}^{N} c_{i j}(x) \int_{-1}^{1} \frac{1}{t-x} \frac{g_{i}(t)}{\left(1-t^{2}\right)^{1 / 2}} d t+\sum_{i=1}^{N} \int_{-1}^{1} K_{i j}(t, x) \frac{g_{i}(t)}{\left(1-t^{2}\right)^{1 / 2}} d t=f_{i}(x), \\
i=1,2, \cdots, N,
\end{aligned}
$$

which results from the system (1.1) if one also considers the behavior (1.2) of the unknown functions $\varphi_{i}(t)(j=1,2, \cdots, N)$, can be reduced, by means of the Gauss-Chebyshev method as it was developed by Erdogan and Gupta [4], to the following system of linear equations:

$$
\begin{aligned}
& \frac{\pi}{n} \sum_{i=1}^{N} c_{i j}\left(x_{r}\right) \sum_{k=1}^{n} \frac{g_{j}}{\left.t_{k}-t_{k}\right)}-\frac{\pi}{n} \sum_{i=1}^{N} \sum_{k=1}^{n} K_{i j}\left(t_{k}, x_{r}\right) g_{i}\left(t_{k}\right)=f_{i}\left(x_{r}\right) \text {, } \\
& i=1,2, \cdots, N, \quad r=1,2, \cdots, n-1,
\end{aligned}
$$

with points $t_{k}$ and $x_{r}$ given by the expressions:

$$
T_{n}\left(t_{k}\right)=0, \quad U_{n-1}\left(x_{r}\right)=0,
$$

where $T_{n}(x)$ and $U_{n-1}(x)$ are the Chebyshev polynomials of the first and second kind respectively, defined by [4]:

$$
T_{n}(x)=\cos n \vartheta, \quad U_{n-1}(x)=\frac{\sin n \vartheta}{\sin \vartheta}, \quad x=\cos \vartheta .
$$

The functions $g_{j}(t)$, apart from satisfying the system of singular integral equations (2.1), are considered as fulfilling particular conditions as well, as in the case of cracks:

$$
\int_{-1}^{1} \frac{g_{j}(t)}{\left(1-t^{2}\right)^{1 / 2}} d t=0, \quad j=1,2, \cdots, N,
$$

from which, by a Gauss-Chebyshev numerical integration, $N$ additional linear equations are produced:

$$
\sum_{k=1}^{n} g_{i}\left(t_{k}\right)=0, \quad j=1,2, \cdots, N .
$$

The system of linear equations (2.2) and (2.6) comprises $(n \times N)$ equations with an equal number of unknowns, and its solution provides the values of the unknown functions $g_{i}(t)$ at points $t_{k}$, determined through the first of Eqs. (2.3).

The difficulty in reducing the system (2.1) into the linear system (2.2) lies in the fact that the singular integrals require special treatment while the ordinary integrals may be approximated by the Gauss-Chebyshev method directly. Erdogan and Gupta [4] thus considered the singular integral

$$
I=\int_{-1}^{1} \frac{1}{t-x} \frac{g(t)}{\left(1-t^{2}\right)^{1 / 2}} d t
$$

written as:

$$
I=\sum_{h=0}^{D} B_{h} \int_{-1}^{1} \frac{1}{t-x} \frac{T_{h}(t)}{\left(1-t^{2}\right)^{1 / 2}} d t,
$$

where the function $g(t)$ was expressed in the form: 


$$
g(t)=\sum_{h=0}^{\mathrm{p}} B_{h} T_{h}(t)
$$

i.e. as the sum of $(p+1)$ Chebyshev polynomials $T_{h}(t)$ of the second kind.

Further, it was taken into account that:

$$
\frac{1}{\pi} \int_{-1}^{1} \frac{1}{t-x} \frac{T_{h}(t)}{\left(1-t^{2}\right)^{1 / 2}} d t=U_{h-1}(x), \quad U_{-1}(x) \equiv 0,
$$

and it was proved that:

$$
\sum_{k=1}^{n} \frac{T_{h}\left(t_{k}\right)}{n\left(t_{k}-x_{r}\right)}=U_{h-1}\left(x_{r}\right), \quad h=0,1, \cdots, n-1, \quad r=1,2, \cdots, n-1,
$$

with points $t_{k}$ and $x_{r}$ defined by relations (2.3). There results the approximate expression of the integral (2.7):

$$
I=\int_{-1}^{1} \frac{1}{t_{k}-x_{r}} \frac{g\left(t_{k}\right)}{\left(1-t_{k}^{2}\right)^{1 / 2 / 2}} d t \simeq \frac{\pi}{n} \sum_{k=1}^{n} \frac{g\left(t_{k}\right)}{t_{k}-x_{r}},
$$

which becomes accurate if $p \leq n-1$ (due to the expression (2.11), which was not proved to be valid for $h>n-1)$, i.e. if $g(t)$ is a polynomial up to $(n-1)$ th degree included.

The approximation (2.12) of the Cauchy-type singular integral $I$ is identical, as far as weights and abscissae are concerned, with the Gauss-Chebyshev method for ordinary integrals, of course under the restriction that the points $x_{r}(r=1,2, \cdots, n-1)$ be given by the second of expressions (2.3). Although the method was considered accurate by Erdogan and Gupta for functions $g(t)$ polynomials up to $(n-1)$ th degree included, nevertheless it is accurate for polynomials up to $2 n$th degree included. It is, therefore, in some ways more accurate than the Gauss-Chebyshev numerical integration method for ordinary integrals:

$$
I=\int_{-1}^{1} \frac{g(t)}{\left(1-t^{2}\right)^{1 / 2}} d t \simeq \frac{\pi}{n} \sum_{k=1}^{n} g\left(t_{k}\right), \quad T_{n}\left(t_{k}\right)=\mathrm{p},
$$

which is accurate for functions $g(t)$ polynomials up to $(2 n-1)$ th degree included.

In order to prove that the Gauss-Chebyshev method for singular integrals of the form (2.7) is accurate for functions $g(t)$ polynomials up to $2 n$th degree included, it is sufficient to prove that relation (2.11) is valid for values of $h$ not only up to $(n-1)$ but up to $2 n$. One notices that for $h=n$ the validity of (2.11) is obvious, considering the determination of the points $t_{k}$ and $x_{r}$ according to relations (2.3). For $(n+1) \leq$ $h \leq 2 n$, in a fashion analogous to that followed by Erdogan and Gupta [4], one considers the following development into simple fractions:

$$
\frac{U_{h-n-1}(x)}{T_{n}(x)}=\sum_{k=1}^{n} \frac{\alpha_{k}}{t_{k}-x}, \quad T_{n}\left(t_{k}\right)=0, \quad 0 \leq h-n-1 \leq n-1,
$$

where:

$$
\alpha_{k}=-\frac{U_{h-n-1}\left(t_{k}\right)}{T_{n}{ }^{\prime}\left(t_{k}\right)}=-\frac{U_{h-n-1}\left(t_{k}\right)}{n U_{n-1}\left(t_{k}\right)} .
$$

By applying the following formula, which can be easily derived from Eqs. (2.4):

$$
U_{h-n-1}(x)=-T_{h}(x) U_{n-1}(x)+T_{n}(x) U_{h-1}(x),
$$

and the following formula from relations (2.3), (2.15) and (2.16): 


$$
\alpha_{k}=\frac{T_{h}\left(t_{k}\right)}{n}, \quad U_{h-n-1}\left(x_{r}\right)=T_{n}\left(x_{r}\right) U_{h-1}\left(x_{r}\right),
$$

then, from relations (2.14), (2.16) and (2.17), the following expression results:

$$
\sum_{k=1}^{n} \frac{T_{h}\left(t_{k}\right)}{n\left(t_{k}-x_{r}\right)}=U_{h-1}\left(x_{r}\right), \quad h=n+1, n+2, \cdots, 2 n .
$$

This is identical with (2.11), but for values of $h$ from $(n+1)$ up to $2 n$ included, because in the fraction on the left-hand side of the series (2.14) the degree of the polynomial of the numerator must be less than the degree of the polynomial of the denominator.

It is thus proved that the numerical expression (2.12) of singular integrals by the Gauss-Chebyshev method is accurate for functions $g(t)$ polynomials of up to $2 n$th degree included. Furthermore, it is obvious that, when using a highly accurate numerical integration method such as the Gauss-Chebyshev method for the approximation of the system (2.1) by means of the linear system (2.2), then the results obtained will in general be very accurate too.

The Gauss-Chebyshev method for ordinary integrals (2.13) was also extended for singular integrals by Chawla and Ramakrishnan [5], who gave the following form of approximation:

$$
\int_{-1}^{1} \frac{1}{t-x} \frac{g(t)}{\left(1-t^{2}\right)^{1 / 2}} d t \simeq \frac{\pi}{n} \sum_{k=1}^{n} \frac{g\left(t_{k}\right)}{t_{k}-x}+\pi g(x) \frac{U_{n-1}(x)}{T_{n}(x)},
$$

which differs from the Gauss-Chebyshev approximate expressions for ordinary integrals only by the second term of its right-hand side, which vanishes in the latter case. This term also vanishes, if the roots $x_{r}(r=1,2, \cdots, n-1)$ of the polynomial $U_{n-1}(x)$ are considered as points $x$. Under this restriction the Gauss-Chebyshev method can be applied to integrals, singular or not, as follows:

$$
I=\int_{-1}^{1} K\left(t, x_{r}\right) g(t) d t \simeq \frac{\pi}{n} \sum_{k=1}^{n} K\left(t_{k}, x_{r}\right) g\left(t_{k}\right)
$$

with kernel:

$$
K(t, x)=\frac{K_{1}(t, x)}{t-x}+K_{2}(t, x),
$$

where $K_{1}(t, x)$ and $K_{2}(t, x)$ are regularly varying functions in relation to both variables within the integration interval $[-1,1]$.

The procedure of Chawla and Ramakrishnan leads to the approximate expression (2.19) through the Gauss-Chebyshev method for singular integrals. This expression can be transformed into (2.12) by selecting points $x_{r}$ according to the second of relations (2.3). It is thus demonstrated that the method of Erdogan and Gupta [4] for the evaluation of Cauchy-type singular integrals of the form (2.7) and called the Gauss-Chebyshev method, is indeed the Gauss-Chebyshev method. Chawla and Ramakrishnan have also shown that the expression (2.19) of a singular integral is accurate for functions $g(t)$ up to $2 n$th degree, included, as has been proved above in a different manner.

3. Advantages of the Lobatto-Chebyshev numerical integration method as compared with the Gauss-Chebyshev method. The above procedure for reducing the system of singular integral equations (2.1) under conditions (2.5) into the system of linear equations 
(2.2) and (2.6) by the Gauss-Chebyshev method leads to the determination of the values of the unknown functions $g_{i}(t)$ at points $t_{k}$, i.e. the roots of the polynomial $T_{n}(x)$, lying within the integration interval $[-1,1]$, but not coinciding with the limits \pm 1 . However, it is exactly at these points that the values of $g_{i}(t)$ are of most interest, as they are proportional to the stress intensity factors at the crack tips, whose determination is the objective of the solution of the system of singular integral equations (2.1). These values $g_{i}(1)$ and $g_{i}(-1)$ may be evaluated by extrapolation, based on the determined values $g_{i}\left(t_{k}\right)$, which were derived from the solution of the system of linear equations, according to the following formulas given by Krenk [6]:

$$
g_{i}( \pm 1)=\frac{1}{n} \sum_{k=1}^{n} U_{2 n-2}\left[\left(\frac{1 \pm t_{k}}{2}\right)^{1 / 2}\right] g_{i}\left(t_{k}\right),
$$

Formulas (3.1) are sufficiently accurate if the function $g_{i}(t)$ is a polynomial of degree not higher than $(n-1)$. Otherwise, significant error can be introduced, which counterbalances the advantages of the Gauss-Chebyshev method with respect to the accuracy of the results obtained, by which a system of singular integral equations can be reduced into a system of linear equations. Besides the error introduced by the use of formulae (3.1), additional error may be generated from the lengthy computations required, in particular when the number of points $n$ is large.

These drawbacks of the Gauss-Chebyshev method as applied to the determination of stress intensity factors, led eventually to the present application of the LobattoChebyshev method, in which the limits \pm 1 of the integration interval are included among the points $t_{k}$. Consequently, no extrapolation is necessary in order to determine the values of the functions $g_{i}(t)$ at these points, i.e. to evaluate the stress intensity factors at the crack tips. However, the Lobatto-Chebyshev method is accurate for integral of the form (2.13) with integrable functions $g(t)$ polynomials up to $(2 n-3)$ rd degree included and not $(2 n-1)$ st degree, as in the Gauss-Chebyshev method. With singular integrals of the form (2.12) the Lobatto-Chebyshev method is accurate for functions $g(t)$ polynomials up to $(2 n-2)$ rd degree included, as will be shown later, and not $2 n$th degree as in the Gauss-Chebyshev method. However, this latter loss of accuracy is not significant, in view of the advantage of not being obliged to use formulae (3.1) for extrapolations which are accurate for functions $g_{i}(t)$ polynomials up to $(n-1)$ th degree only.

Nevertheless, it must be mentioned here that one might be interested in the values of the unknown functions along the whole of the integration interval $[-1,1]$ and not only for the determination of stress intensity factors. In this case, the unknown functions are expressed by means of formulae of the form (2.9), where the coefficients $B_{h}$ are given by formulae of the following form [6]:

$$
B_{h}=\frac{2}{n} \sum_{k=1}^{n} T_{h}\left(t_{k}\right) g\left(t_{k}\right), \quad h=1,2, \cdots, n-1, \quad B_{0}=\frac{1}{n} \sum_{k=1}^{n} g\left(t_{k}\right)
$$

and the Gauss-Chebyshev method should be applied, rather than the Lobatto-Chebyshev one, although formulas analogous to (3.2) may be easily derived for the latter as well.

For ordinary integrals, the Lobatto-Chebyshev method assumes the form [7]:

$$
\begin{aligned}
& I=\int_{-1}^{1} \frac{g(t)}{\left(1-t^{2}\right)^{1 / \overline{2}}} d t=\frac{\pi}{n-1}\left[\frac{1}{2} g(-1)+\frac{1}{2} g(+1)+\sum_{k=2}^{n-1} g\left(t_{k}\right)\right], \\
& U_{n-2}\left(t_{k}\right)=0, \quad k=2,3, \cdots, n-1,
\end{aligned}
$$


which is accurate for functions $g(t)$ polynomials up to $(2 n-3)$ rd degree, as already stated.

The authors are not familiar with any applications of the Lobatto-Chebyshev method to singular integrals of the form (2.7) like those of the Gauss-Chebyshev method. Hence the procedure will be developed below.

4. The Lobatto-Chebyshev numerical integration method as applied to the evaluation of singular integrals. The procedure to be followed in the present section is similar to that applied by Erdogan and Gupta [4] to the Gauss-Chebyshev method. Let the following expansion into simple fractions be considered:

$$
\frac{T_{n-h-1}(x)}{\left(1-x^{2}\right) U_{n-2}(x)}=\sum_{k=1}^{n} \frac{\alpha_{k}}{t_{k}-x}, \quad\left(1-t_{k}^{2}\right) U_{n-2}\left(t_{k}\right)=0,
$$

where

$$
\alpha_{k}=-\frac{T_{n-h-1}\left(t_{k}\right)}{\left[\left(1-t_{k}^{2}\right) U_{n-2}\left(t_{k}\right)\right]^{\prime}} .
$$

In the expansion (4.1) of the fraction on the left-hand side, the degree of the polynomial of the numerator must be less than the degree of the polynomial of the denominator, i.e.:

$$
0 \leq n-h-1 \leq n-1 \text { or } 0 \leq h \leq n-1 .
$$

By considering formulae (2.4), defining the Chebyshev polynomials $T_{n}(x)$ and $U_{n-1}(x)$, one can easily show that:

$$
\left[\left(1-x^{2}\right) U_{n-2}(x)\right]^{\prime}=-x U_{n-2}(x)-(n-1) T_{n-1}(x)
$$

and also that:

$$
T_{n-h-1}(x)=T_{h}(x) T_{n-1}(x)+U_{h-1}(x)\left(1-x^{2}\right) U_{n-2}(x), \quad h \geq 0,
$$

when, from expressions (4.2) for the coefficients $\alpha_{k}$, one obtains:

$\alpha_{1}=\frac{T_{h}(-1)}{2(n-1)}, \quad \alpha_{n}=\frac{T_{h}(1)}{2(n-1)}, \quad \alpha_{k}=\frac{T_{h}\left(t_{k}\right)}{n-1}, \quad k=2,3, \cdots, n-1$,

taking into account that:

$$
t_{1}=-1, \quad t_{n}=1, \quad U_{n-2}\left(t_{k}\right)=0, \quad k=2,3, \cdots, n-1,
$$

as well as that:

$$
T_{n-1}(1)=1, \quad T_{n-1}(-1)=(-1)^{n-1}, \quad U_{n-2}(1)=n-1, \quad U_{n-2}(-1)=(-1)^{n}(n-1) .
$$

The series expansion (4.1) now assumes the form:

$$
\begin{aligned}
\sum_{k=1}^{n} \lambda_{k} \frac{T_{h}\left(t_{k}\right)}{(n-1)\left(t_{k}-x\right)}=U_{h-1}+\frac{T_{h}(x) T_{n-1}(x)}{\left(1-x^{2}\right) U_{n-2}(x)}, \\
\lambda_{k}=\left\{\begin{array}{l}
1 / 2, k=1, n \\
1, k=2,3, \cdots, n-1 .
\end{array}\right.
\end{aligned}
$$

By selecting points $x_{r}$ from:

$$
T_{n-1}\left(x_{r}\right)=0, \quad r=1,2, \cdots, n-1,
$$


and by applying expansion (4.9) for these, one obtains:

$$
\sum_{k=1}^{n} \lambda_{k} \frac{T_{h}\left(t_{k}\right)}{(n-1)\left(t_{k}-x_{r}\right)}=U_{h-1}\left(x_{r}\right), U_{-1}(x) \equiv 0, \quad h=0,1, \cdots, n-1,
$$

which is valid for $h$ from 0 up to $(n-1)$ included.

For $h$ from $(n-1)$ up to $(2 n-2)$, one can prove that the expansion (4.11) is also valid, provided that, instead of the original expansion (4.1), one considers the expansion:

$$
\frac{T_{h-n+1}(x)}{\left(1-x^{2}\right) U_{n-2}(x)}=\sum_{k=1}^{n} \frac{\alpha_{k}}{t_{k}-x}, \quad\left(1-t_{k}^{2}\right) U_{n-2}\left(t_{k}\right)=0 .
$$

Consequently, the expansion (4.11) is valid for $0 \leq h \leq(2 n-2)$, while the corresponding expansion (2.18) for the Gauss-Chebyshev method is valid for $0 \leq h \leq 2 n$.

By comparing Eqs. (2.10) and (4.11) and taking into account that a polynomial $g(t)$ of $p$ th degree can be expanded into a series of Chebyshev polynomials, as in Eq. (2.9), one can develop the following numerical integration method for singular integrals:

$$
\begin{aligned}
I=\int_{-1}^{1} \frac{1}{t_{k}}-\bar{x}_{r} \frac{g\left(t_{k}\right)}{\left(1-t_{k}^{2}\right)^{1 / 2}} d t \simeq \frac{\pi}{n-1} \sum_{k=1}^{n} \lambda_{k} \frac{g\left(t_{k}\right)}{t_{k}-x_{r},} \\
\lambda_{k}=\left\{\begin{array}{l}
1 / 2, k=1, n \\
1, k=2,3, \cdots, n-1
\end{array}\right.
\end{aligned}
$$

which is accurate for functions $g(t)$ polynomials up to $(2 n-2)$ th degree included. Relation (4.13) is identical, as far as weights and abscissae are concerned, with the Lobatto-Chebyshev method for ordinary integrals (3.3) and it can be considered as an extension of the latter for singular integrals. Of course points $x_{r}$ are not arbitrary, but they are given from Eq. (4.10). Also, one may note that the Lobatto-Chebyshev method for ordinary integrals (3.3) is accurate for functions $g(t)$ polynomials up to $(2 n-3)$ th degree, while for singular integrals (4.13) is accurate for functions $g(t)$ polynomials up to $(2 n-2)$ th degree.

By applying the Lobatto-Chebyshev method to the system (2.1) under the conditions (2.5), the following system of linear equations results:

$$
\begin{array}{r}
\frac{\pi}{n-1} \sum_{i=1}^{N} c_{i j}\left(x_{r}\right) \sum_{k=1}^{n} \lambda_{k} \frac{g_{i}\left(t_{k}\right)}{t_{k}-x_{r}}+\frac{\pi}{n-1} \sum_{i=1}^{N} \sum_{k=1}^{n} \lambda_{k} K_{i j}\left(t_{k}, x_{r}\right) g_{i}\left(t_{k}\right)=f_{i}\left(x_{r}\right), \\
\sum_{k=1}^{n} \lambda_{k} g_{j}\left(t_{k}\right)=0, \quad \lambda_{k}=\left\{\begin{array}{l}
1 / 2, k=1, n \\
1, k=2,3, \cdots, n-1,
\end{array}\right.
\end{array}
$$

which consists of $(n \times N)$ equations and an equal number of unknowns, as is the case with the system (2.2) and (2.6) derived by the Gauss-Chebyshev method.

One can also note that the Lobatto-Chebyshev method for singular integrals can be developed in a way similar to that followed by Chawla and Ramakrishnan [5] with the Gausi-Chebyshev method (as well as the Gauss-Jacobi method).

5. Application to the determination of the stress intensity factors at the tips of a cruciform crack. As an application of the above Gauss-Chebyshev and LobattoChebyshev methods, the problem of a cruciform crack in an infinite isotropic elastic medium under constant load $\sigma$ along all its four branches is to be treated (Fig. 1). Such a crack can be considered as a system of two cracks of length $2 \alpha$ intersecting each other 


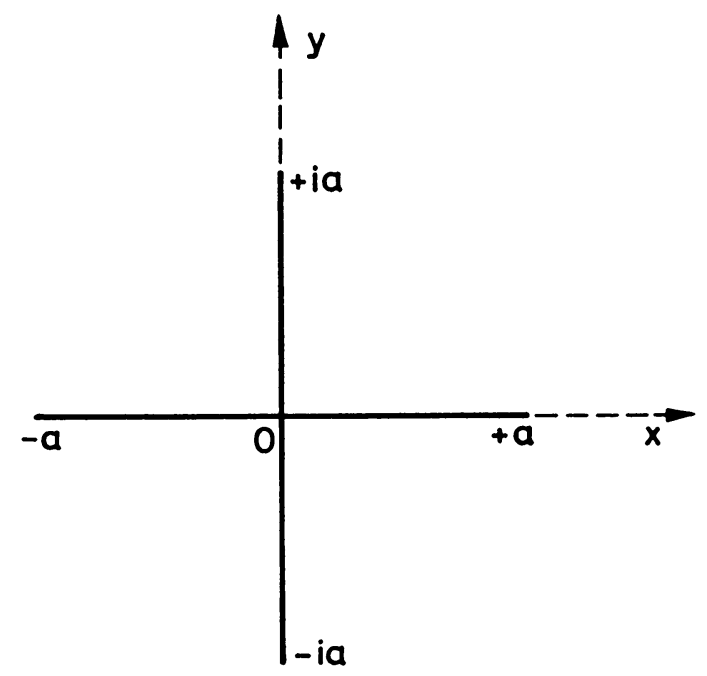

FIg. 1. Cruciform crack in an infinite medium.

at right angles, in which case the Datsyshin and Savruk [2] method is applicable. The problem can thus be reduced into a singular integral equation, which must be valid for both cracks, under the given conditions of symmetry in geometry and loading of the latter. After using the dimensionless length variables $t$ and $x$, there follows the equation:

$$
\frac{1}{\pi} \int_{-1}^{1}\left[\frac{1}{t-x}+\frac{t\left(t^{2}-x^{2}\right)}{\left(t^{2}+x^{2}\right)^{2}}\right] \varphi(t) d t=\sigma,
$$

where the unknown function $\varphi(t)$ is proportional to the dislocation density along the crack branches. This exhibits a singularity of order $(-1 / 2)$ at the crack tips; it may thus assume the form:

$$
\varphi(t)=\frac{g(t)}{\left(1-t^{2}\right)^{1 / 2}}
$$

with function $g(t)$ regularly varying along the crack branches. One should also note that both functions $\varphi(t)$ and $g(t)$ are odd because of the opposite sense of deformation of each branch of the intersecting cracks. Then Eq. (5.1), because of Eq. (5.2), can be approximated by the following system of linear equations:

$$
\begin{aligned}
\frac{1}{n} \sum_{k=1}^{n}\left[\frac{t_{k}}{t_{k}^{2}-x_{r}{ }^{2}}+\frac{t_{k}\left(t_{k}{ }^{2}-x_{r}{ }^{2}\right)}{\left(t_{k}^{2}+x_{r}^{2}\right)^{2}}\right] g\left(t_{k}\right)=\sigma, & r=1,2, \cdots, n, \\
t_{k}, x_{r} \geq 0, & T_{2 n}\left(t_{k}\right)=0, \quad U_{2 n-1}\left(x_{r}\right)=0,
\end{aligned}
$$

if the Gauss-Chebyshev method for $2 n$ points be applied, or by the system:

$$
\begin{aligned}
\frac{1}{n-1} \sum_{k=1}^{n} \lambda_{k}\left[\frac{t_{k}}{t_{k}{ }^{2}-x_{r}{ }^{2}}+\frac{t_{k}\left(t_{k}{ }^{2}-x_{r}{ }^{2}\right)}{\left(t_{k}{ }^{2}+x_{r}{ }^{2}\right.}\right] g\left(t_{k}\right)=\sigma, \quad r=1,2, \cdots, n, & \\
t_{k}, x_{r} \geq 0, \quad\left(1-t_{k}{ }^{2}\right) U_{2 n-2}\left(t_{k}\right) & =0, \quad T_{2 n-1}\left(x_{r}\right)=0,
\end{aligned}
$$


if the Lobatto-Chebyshev method for $2 n$ points be applied. Note that the condition of single-valuedness of displacements is automatically satisfied, due to the symmetry of geometry and loading of the cruciform crack.

The systems (5.3) and (5.4) have been solved for various values of $n$ and the value $g(1)$ of the unknown function $g(t)$ for $t=1$ was determined both by extrapolation following the application of the Gauss-Chebyshev method and directly from the solution of system (5.4) according to the Lobatto-Chebyshev method. The stress intensity factor $k_{I}$ at the tips of all four branches of the cruciform crack of Fig. 1 can be determined in a straightforward manner from the value $g(1)$ of the unknown function $g(t)$ for $t=1$ from the expression:

$$
k_{I}=\sigma \alpha^{1 / 2} g(1) .
$$

The results are given in Table 1 , and it is obvious that, with increasing $n$, they con-

TABLE 1. Reduced values of the stress intensity factor at the tips of a cruciform crack.

\begin{tabular}{ccc}
\hline Method & Gauss-Chebyshev & Lobatto-Chebyshev \\
\hline$n$ & \multicolumn{1}{c}{$k_{I} /\left(\sigma \alpha^{1 / 2}\right)=g(1)$} \\
\hline 2 & 0.94445 & 0.83658 \\
3 & 0.83635 & 0.85970 \\
4 & 0.83882 & 0.86387 \\
5 & 0.86289 & 0.86449 \\
6 & 0.86381 & 0.86441 \\
7 & 0.86528 & 0.86424 \\
8 & 0.86282 & 0.86408 \\
9 & 0.86503 & 0.86396 \\
10 & 0.86283 & 0.86387 \\
11 & 0.86464 & 0.86380 \\
\hline
\end{tabular}

verge much faster and more smoothly by the Lobatto-Chebyshev method than by the Gauss-Chebyshev method towards the limit 0.8636 , which is approximately the correct value of the reduced stress intensity factor as derived by Rooke and Sneddon [8]. The fact that the value of the reduced stress intensity factor does not converge as fast as one might expect with increasing $n$ by both methods (particularly by the Gauss-Chebyshev method) is due to the discontinuity of the derivatives of the unknown function $g(t)$ for $t=0$, i.e. at the center of the cruciform crack.

\section{REFERENCES}

[1] R. V. Gol'dshtein and L. N. Savova, Determination of crack opening and stress intensity coefficients for a smooth curvilinear crack in an elastic plane, Mech. Solids 7, 64-71 (1972) (translation of: Izv. AN SSSR, Mekh. Tverdogo Tela 7, 69-78 (1972))

[2] A. P. Datsyshin and M. P. Savruk, A system of arbitrarily oriented cracks in elastic solids, J. Appl. Math. Mech. (PMM) 37, 306-313 (1973) (translation of: Prik. Mat. Mekh. 37, 326-332 (1973))

[3] F. Erdogan, G. D. Gupta and T. S. Cook, Numerical solution of singular integral equations, in Methods of analysis and solutions of crack problems (Vol. 1 in the series Mechanics of fracture, edited by G. C. Sih), Noordhoff Int. Publ., Leyden, 1973, pp. 368-425

[4] F. Erdogan and G. D. Gupta, On the numerical solution of singular integral equations, Quart. Appl. Math. 30, 525-534 (1972) 
[5] M. M. Chawla and T. R. Ramakrishnan, Modified Gauss-Jacobi quadrature formulas for the numerical evaluation of Cauchy-type singular integrals, BIT 14, 14-21 (1974)

[6] S. Krenk, On the use of the interpolation polynomial for solutions of singular integral equations, Quart. Appl. Math. 32, 479-484 (1975)

[7] Z. Kopal, Numerical analysis, Chapman and Hall, London, 1961

[8] D. P. Rooke and I. N. Sneddon, The crack energy and the stress intensity factor for a cruciform crack deformed by internal pressure, Int. J. Engng. Science 7, 1079-1089 (1969) 